\title{
Etiology and pathogenesis of the Marfan syndrome: current understanding
}

\author{
Reed E. Pyeritz \\ Departments of Medicine and Genetics, Perelman School of Medicine at the University of Pennsylvania, Philadelphia, PA, USA \\ Correspondence to: Reed E. Pyeritz, MD, PhD. William Smilow Professor of Medicine, 11-133 Smilow Center for Translational Research, 3400 Civic \\ Center Blvd., Philadelphia, PA 19104, USA. Email: reed.pyeritz@uphs.upenn.edu.
}

\begin{abstract}
Much has changed regarding Marfan syndrome (MFS) over the past few decades. Once described solely as a heritable disorder of connective tissue, MFS is now one of a number of conditions recognized to be a disorder of abnormal signalling in the TGF- $\beta$ pathway. The cardinal features of MFS, once encompassed by the ocular, skeletal and cardiovascular systems, are now known to encompass many more organ systems, especially as people with MFS grow older. They are growing older by several decades compared to the 1970's because of profound improvements in diagnosis and management of the cardiovascular features, especially dilatation of the aortic root. This dilatation can be detected first in infancy and followed up by echocardiography. Progressive enlargement increases the risk of type A dissection and aortic regurgitation, the major causes of early mortality, in untreated patients today. Medical therapy with $\beta$-adrenergic blockade, first shown to be effective in the 1980's, can retard this dilatation. In the past decade, angiotensin receptor blockade, which reduces aberrant signalling through one of the TGF- $\beta$ pathways, also can be effective. However, when dilatation of the root becomes such that the risk of dissection increases to an unacceptable degree, surgical therapy becomes necessary. In the mid-1970's, the composite graft, introduced by Hugh Bentall, markedly reduced mortality. In the past decade, a valve-spring aortic root replacement, advanced by Tirone David, has become widely adopted. Mid-term results are quite encouraging. Other cardiovascular involvement, such as mitral valve prolapse, type B dissection, and dilatation and dissection of aortic branches, also require close monitoring. Currently, life-expectancy in people with MFS who are diagnosed early and treated prophylactically is approaching that of the general population.
\end{abstract}

Keywords: Aortic aneurysm; beta-blockade; TGF- $\beta$

Submitted May 25, 2017. Accepted for publication Aug 31, 2017.

doi: $10.21037 /$ acs.2017.10.04

View this article at: http://dx.doi.org/10.21037/acs.2017.10.04

I arrived at the Johns Hopkins Hospital to begin my senior residency in medicine and medical genetics fellowship still wearing scrubs, having been on-call at the Peter Bent Brigham the night before. Within 30 minutes, Victor McKusick, the then chief of medicine but also one of the founders of the discipline of medical genetics, called me into his office to meet a young adolescent with Marfan syndrome (MFS). The boy's father had recently suffered a type A aortic dissection, and had been saved by Vincent Gott, who performed the first Bentall procedure that he, or anyone at Hopkins, had ever attempted. I wound up caring for the boy and his extended family for 16 years, altering my management as knowledge evolved. The boy was tall and lanky and had an aortic root dimension at the upper limit of normal. The same was true for both of his sisters, who Victor and I also decided had MFS, and treated them accordingly. Of course, we were nearly a decade and a half from discovering the cause of MFS, so molecular genetic testing to prove the diagnoses was impossible.

MFS was one of the seven heritable disorders of connective tissue included in Dr. McKusick's seminal monograph of the same name (1), first published in 1956. In fact, in 1955, he was the first to label MFS, or any condition, a heritable disorder of connective tissue (2). 
Notions of pathogenesis had not really evolved in the subsequent 16 years, as I was educated about MFS by reading the 4th edition of that book. The connective tissue was "weak", which accounted for the dilatation of the sinuses of Valsalva, aortic dissection, dislocation of the lens, scoliosis, and pneumothoraces. Of course, why the long bones overgrew was not easily explained, but that did not dissuade anyone from the notion of a structural defect in the extracellular matrix (3). The so-called "cystic medial necrosis" of the dilated aortic wall was assumed to represent the on-going damage and the body's attempt at repair. This histopathologic characterization always confounded me since there were neither cysts nor necrosis present. Eventually, the term "medial degeneration" emerged, even if the underlying injury-repair process remained accepted.

Over several decades, a variety of components of the extracellular matrix were "identified" biochemically as being abnormal, including several forms of collagen, production of hyaluronic acid and defects in elastin. What was clear to most clinical investigators over the latter half of the twentieth century was that efforts at treatment would best be stimulated by pathogenesis rather than etiology.

Since hypertension was then a well-accepted association with acute aortic dissection in the general population, it made sense to control the blood pressure of people with MFS. However, studies of turkeys that were prone to aortic rupture, either spontaneously or because of treatment with $\beta$-aminopropionitrile (4) [and eventually diethylstilbestrol (5)], suggested a nuance that informed the first therapeutic trial in human MFS. Treatment of the turkeys with the first drug approved for humans in a new class of medications, the $\beta$-adrenergic blocking drug, propranolol, that reduced their blood pressure if they were hypertensive, or did not alter their blood pressure if they were naturally hypotensive, reduced the incidence of aortic rupture (4). In the latter case, the beneficial effect was felt to be a reduction in $\mathrm{dP} / \mathrm{dt}$. Propranolol was shown to protect the aorta from rupture in the blotchy mouse as well (6). Propranolol was first used in a few patients with MFS in 1971, with little effect, probably because their proximal aortas were already substantially dilated (7). But working under the hypothesis that the connective tissue architecture of the aortic wall, even when minimally dilated, was "weak", we began a randomized, non-blinded trial in adolescents and young adults. The doses used to treat the subjects were optimized based on reduction of exercise heart rate and $\mathrm{dP} / \mathrm{dt}$ measured from $\mathrm{M}$-mode echocardiograms. After a decade, the results showed both a reduction in complications and in the rate of aortic root dilatation in the treated patients (8). The benefits of $\beta$-blockade were replicated in most, but not all, subsequent studies. What was clear was the need to start treatment before substantial aortic root dilatation was present. Propranolol, and quickly newer $\beta$-blockers, became, in most clinics, the standard medical treatment.

Fast forward to 1991 when a pediatric cardiology fellow, Hal Dietz, collaborated with me and other colleagues at Hopkins first, to precisely map the chromosomal locus of the "Marfan gene" (9), and second, to determine that the product of that gene (FBN1) was fibrillin-1, an intrinsic component of the extracellular microfibril, which, in turn, was present in all elastic fibers, was mutated (10). This finding also supported the notion of "weak connective tissue". Additionally, a number of other investigators contributed greatly to understanding the etiology. Lynn Sakai and Eva Engvall first defined fibrillin as a component of elastic fibers (11). Lena Peltonen and her group first mapped the gene for MFS to human chromosome 15 (12). Dianna Milewicz, then a fellow with Peter Byers, showed that fibrillin was defective in cell cultures of fibroblasts from people with MFS (13). David Hollister and colleagues demonstrated immunohistopathologic abnormalities of fibrillin in skin biopsies and dermal cell cultures from patients (14). The gene for fibrillin-1 is relatively large, encompassing 65 exons and nearly 10,000 nucleotides. Subsequently, over one thousand mutations in FBN1 have been identified to cause MFS (15). Interestingly, mutations in FBN1 have been found to cause a variety of other disorders, including forms of dwarfism, the antithesis of MFS (16).

However, a few years later, the Dietz lab capitalized on the fact that a fundamental function of fibrillin- 1 was to bind the latent transforming growth factor- $\beta$ binding protein (LTGBP). As a result, control of the cellular activity of the growth factor was disrupted. Defective fibrillin-1 resulted in excessive activity of transforming growth factor- $\beta$ (TGF- $\beta$ ), which led to a cascade through its canonical signaling pathway, resulting in increased activity of SMAD2/3. The first proof that excessive TGF- $\beta$ signaling is present in affected tissue, in mice bearing a pathogenic human mutation in FBN1, occurred in lung tissues, in which the terminal alveolar septae failed to mature (17). This is likely the reason patients with MFS are susceptible to pneumothoraces. Subsequently, the same phenomenon was demonstrated in mitral valve prolapse in the same mouse strain (18). These early experiments clearly 
demonstrated that some manifestations of MFS, at least in mice, were not due to "weak connective tissue".

Losartan, an AT1 angiotensin receptor blocking drug, was shown to protect mouse models of MFS from developing aortic aneurysms (19). Not only did the aorta not dilate or dissect, but the histopathology appeared unaffected. Overexpression of TGF- $\beta$ was shown to result in several of the biochemical findings once thought to be primary, such as increased hyaluronan deposition and expression of matrix metalloproteinases. Treatment of the same mice with $\beta$-blockade had a modest effect of aortic dilatation without affecting histopathology (19). This fresh insight into pathophysiology promptly led to a suggestion for a clinical trial of losartan in human Marfan patients. After much negotiation with the National Heart, Lung and Blood Institute and the National Marfan Foundation, and through the Pediatric Heart Network, a trial was designed. Unfortunately, patients were resistant to a placebo, so the trial was two-armed, with losartan being compared to atenolol in a double-blind manner. The trial was limited to subjects less than 24 years old, who had an aortic Z-score of 3 or greater. Thus, their aortic roots already had at least moderate dilatation. The doses of each drug were optimized. After 3 years of treatment of 604 randomized subjects, there was no significant difference between the two groups. In fact, $\beta$-blockade was slightly more effective (20). Younger subjects tended to benefit more with either drug. Additional randomized trials have tended to confirm these results; several have compared mono-therapy with combination therapy, with mixed results (21).

The canonical pathway of TGF- $\beta$ signaling is through the angiotensin- 1 receptor. Thus, neutralization of TGF- $\beta$ or antagonism of AT1 were expected to be equally effective in preventing aortic disease in the mouse model. However, antagonism of AT1 with losartan was more effective than using a TGF- $\beta$ antibody (22). In fact, early inhibition of TGF- $\beta$ proved detrimental in mice, suggesting that such treatment should be avoided at some early stage in humans.

Further studies focusing on the role of increased TGF- $\beta$ have focused on both the canonical signaling pathway (through the SMAD2/3 cascade) and noncanonical (nonSMAD) pathway (involving ERK1/2 and other mediators).

From the early days of treatment, some patients could not tolerate $\beta$-blockade, and for years calcium-channel blockade was recommended as an alternative. Surprisingly, first in the mouse model treated with either amlodipine or verapamil, and subsequently in retrospective analysis of humans, calcium-channel blockade was found to be harmful, probably through protein kinase $\mathrm{C}$ (PKC) activation (23). Interestingly, hydralazine inhibits $\mathrm{PKC}$ and might be an alternative therapy for those patients who cannot tolerate $\beta$-blockade or AT1 blockade. These findings have greatly altered medical treatment of human MFS.

Further understanding of the pathogenesis of MFS, especially in the human, will undoubtedly lead to more effective medical treatments in the future. For example, inducible nitric oxide synthase (NOS2) was recently shown to stimulate aortic aneurysm formation in mice with MFS of in those deficient in the metalloprotease ADAMTS1 (24). Inactivation of NOS2 inhibited the development of aortic pathology in both strains of mice. Inhibitors of NOS2 deserve study as potential protectors of aortic aneurysm in humans.

Of course, to date, nothing has benefited people with MFS (and the many other forms of heritable disorders of the aortic root) more than the introduction and evolution of prophylactic surgical repair, first with the composite graft (25), and then with valve-sparing root repair (26).

In an ironic turn of events, the family that Victor introduced to me my first day at Johns Hopkins, has done very well, being followed as having MFS. However, when molecular genetic analysis was performed recently, their mutation is in SMAD3.

\section{Acknowledgements}

None

\section{Footnote}

Conflicts of Interest: The author has no conflicts of interest to declare.

\section{References}

1. McKusick VA. Heritable Disorders of Connective Tissue. 1st edition. St. Louis: C.V. Mosby Company, 1956.

2. McKusick VA. The cardiovascular aspects of Marfan's syndrome: a heritable disorder of connective tissue. Circulation 1955;11:321-42.

3. Pyeritz RE, McKusick VA. The Marfan syndrome: diagnosis and management. N Engl J Med 1979;300:772-7.

4. Simpson CF, Kling JM, Palmer RF. The use of propranolol for the protection of turkeys from the development of beta-aminopropionitrile-induced aortic ruptures. Angiology 1968;19:414-8. 
5. Simpson CF. Relation of hemodynamics to the incidence of diethylstilbestrol-induced aortic ruptures in hypertensive and hypotensive lines of turkeys. Atherosclerosis 1978;30:249-54.

6. Brophy C, Tilson JE, Tilson MD. Propranolol delays the formation of aneurysms in the male blotchy mouse. J Surg Res 1988;44:687-9.

7. Halpern BL, Char F, Murdoch JL, et al. A prospectus on the prevention of aortic rupture in the Marfan syndrome with data on survivorship without treatment. Johns Hopkins Med J 1971;129:123-9.

8. Shores J, Berger KR, Murphy EA, et al. Progression of aortic dilatation and the benefit of long-term betaadrenergic blockade in Marfan's syndrome. N Engl J Med 1994;330:1335-41.

9. Dietz HC, Pyeritz RE, Hall BD, et al. The Marfan syndrome locus: confirmation of assignment to chromosome 15 and identification of tightly linked markers at 15q15-q21.3. Genomics 1991;9:355-61.

10. Dietz HC, Cutting GR, Pyeritz RE, et al. Marfan syndrome caused by a recurrent de novo missense mutation in the fibrillin gene. Nature 1991;352:337-9.

11. Sakai LY, Keene DR, Engvall E. Fibrillin, a new 350-kD glycoprotein, is a component of extracellular microfibrils. J Cell Biol 1986;103:2499-509.

12. Kainulainen K, Pulkkinen L, Savolainen A, et al. Location on chromosome 15 of the gene defect causing Marfan syndrome. N Engl J Med 1990;323:935-9.

13. Milewicz DM, Pyeritz RE, Crawford ES, et al. Marfan syndrome: defective synthesis, secretion, and extracellular matrix formation of fibrillin by cultured dermal fibroblasts. J Clin Invest 1992;89:79-86.

14. Hollister DW, Godfrey M, Sakai LY, et al. Immunohistologic abnormalities of the microfibrillarfiber system in the Marfan syndrome. N Engl J Med 1990;323:152-9.

15. Faivre L, Collod-Beroud G, Loeys BL, et al. Effect of mutation type and location on clinical outcome in 1,013 probands with Marfan syndrome or related phenotypes and FBN1 mutations: an international study. Am J Hum Genet 2007;81:454-66.

Cite this article as: Pyeritz RE. Etiology and pathogenesis of the Marfan syndrome: current understanding. Ann Cardiothorac Surg 2017;6(6):595-598. doi: 10.21037/acs.2017.10.04
16. Sakai LY, Keene DR, Renard M, et al. FBN1: The diseasecausing gene for Marfan syndrome and other genetic disorders. Gene 2016;591:279-91.

17. Neptune ER, Frischmeyer PA, Arking DE, et al. Dysregulation of TGF-beta activation contributes to pathogenesis in Marfan syndrome. Nat Genet 2003;33:407-11.

18. Ng CM, Cheng A, Myers LA, et al. TGF-beta-dependent pathogenesis of mitral valve prolapse in a mouse model of Marfan syndrome. J Clin Invest 2004;114:1586-92.

19. Habashi JP, Judge DP, Holm TM, et al. Losartan, an AT1 antagonist, prevents aortic aneurysm in a mouse model of Marfan syndrome. Science 2006;312:117-21.

20. Lacro RV, Dietz HC, Sleeper LA, et al. Atenolol versus losartan in children and young adults with Marfan's syndrome. N Engl J Med 2014;371:2061-71.

21. Singh MN, Lacro RV. Recent Clinical Drug Trials Evidence in Marfan Syndrome and Clinical Implications. Can J Cardiol 2016;32:66-77.

22. Cook JR, Clayton NP, Carta L, et al. Dimorphic effects of transforming growth factor- $\beta$ signaling during aortic aneurysm progression in mice suggest a combinatorial therapy for Marfan syndrome. Arterioscler Thromb Vasc Biol 2015;35:911-7.

23. Doyle JJ, Doyle AJ, Wilson NK, et al. A deleterious geneby-environment interaction imposed by calcium channel blockers in Marfan syndrome. Elife 2015;4. pii: e08648.

24. Oller J, Méndez-Barbero N, Ruiz EJ, et al. Nitric oxide mediates aortic disease in mice deficient in the metalloprotease Adamts1 and in a mouse model of Marfan syndrome. Nat Med 2017;23:200-12.

25. Gott VL, Pyeritz RE, Magovern GJ Jr, et al. Surgical treatment of aneurysms of the ascending aorta in the Marfan syndrome. Results of composite-graft repair in 50 patients. N Engl J Med 1986;314:1070-4.

26. Song HK, Preiss LR, Maslen CL, et al. Valve-sparing aortic root replacement in patients with Marfan syndrome enrolled in the National Registry of Genetically Triggered Thoracic Aortic Aneurysms and Cardiovascular Conditions. J Heart Valve Dis 2014;23:292-8. 